\title{
Hyperspectral multiplex single- particle tracking of different receptor subtypes labeled with quantum dots in live neurons
}

Simon Labrecque

Jean-Philippe Sylvestre

Stephane Marcet

Francesca Mangiarini

Brice Bourgoin

Marc Verhaegen

Sébastien Blais-Ouellette

Paul De Koninck 


\title{
Hyperspectral multiplex single-particle tracking of different receptor subtypes labeled with quantum dots in live neurons
}

\author{
Simon Labrecque, ${ }^{\mathrm{a}}$ Jean-Philippe Sylvestre, ${ }^{\mathrm{b}}$ Stephane Marcet, ${ }^{\mathrm{b}}$ Francesca Mangiarini, ${ }^{\mathrm{b}}$ Brice Bourgoin, ${ }^{\mathrm{b}}$ \\ Marc Verhaegen, ${ }^{b}$ Sébastien Blais-Ouellette, ${ }^{b, *}$ and Paul De Koninck ${ }^{\mathrm{a}, \mathrm{c}, *}$ \\ anstitut Universitaire en santé mentale de Québec, 2601 Chemin de la Canardière, Québec G1J 2G3, Canada \\ ${ }^{b}$ Photon Etc., 5795 de Gaspé ave., Montréal, Québec H2S 2X3, Canada \\ 'Université Laval, Department of Biochemistry, Microbiology, and Bio-informatics, Québec G1K 7P4, Canada
}

\begin{abstract}
The efficacy of existing therapies and the discovery of innovative treatments for central nervous system (CNS) diseases have been limited by the lack of appropriate methods to investigate complex molecular processes at the synaptic level. To improve our capability to investigate complex mechanisms of synaptic signaling and remodeling, we designed a fluorescence hyperspectral imaging platform to simultaneously track different subtypes of individual neurotransmitter receptors trafficking in and out of synapses. This imaging platform allows simultaneous image acquisition of at least five fluorescent markers in living neurons with a high-spatial resolution. We used quantum dots emitting at different wavelengths and functionalized to specifically bind to single receptors on the membrane of living neurons. The hyperspectral imaging platform enabled the simultaneous optical tracking of five different synaptic proteins, including subtypes of glutamate receptors (mGluR and AMPAR) and postsynaptic signaling proteins. It also permitted the quantification of their mobility after treatments with various pharmacological agents. This technique provides an efficient method to monitor several synaptic proteins at the same time, which could accelerate the screening of effective compounds for treatment of CNS disorders. (C) The Authors. Published by SPIE under a Creative Commons Attribution 3.0 Unported License. Distribution or reproduction of this work in whole or in part requires full attribution of the original publication, including its DOI. [DOI: 10.1117/1.JBO.21.4.046008]
\end{abstract}

Keywords: nanoparticles; synaptic receptors; single-molecule tracking; hippocampal cultures; hyperspectral imaging.

Paper 150551PRRR received Aug. 17, 2015; accepted for publication Mar. 15, 2016; published online Apr. $25,2016$.

\section{Introduction}

Synaptic transmission and plasticity involve a plethora of different molecules and proteins. Understanding synaptic function at the molecular level is a crucial step in the discovery and development of compounds for treating neurological, neurodegenerative, and psychiatric diseases. To accelerate such discoveries, the capacity to monitor several molecules in parallel would be highly valuable. Imaging studies have demonstrated that synaptic proteins are extremely mobile between extrasynaptic and synaptic domains. ${ }^{1}$ Thus, being able to track the spatial dynamics of several proteins on the same sample becomes essential. Optical methods based on fluorescence microscopy enable the investigation of specific molecules at low concentration with a good signal-to-noise ratio, either in fixed or living specimen. However, due to spectral overlap, conventional fluorescence imaging techniques are usually limited to a maximum of three fluorophores.

Hyperspectral fluorescence imaging can increase the capability to discriminate additional fluorophores simultaneously since it detects photons emitted over many narrow spectral bands. ${ }^{2}$ Important factors for spectral filtering systems include the degree of transmission, wavelength tuning range, bandwidth, tuning speed, and out-of-band blocking power. ${ }^{3}$ Several hyperspectral

\footnotetext{
*Address all correspondence to: Sébastien Blais-Ouellette, E-mail: sbo@ photonetc.com; Paul De Koninck, E-mail: paul.dekoninck@neurosciences. ulaval.ca
}

filtering systems covering the visible spectrum are already available for microscopy systems, such as acousto-optic tunable filters (AOTF), ${ }^{4}$ liquid crystal tunable filters $(\mathrm{LCTF}),{ }^{5}$ or thin-film tunable filters (TFTF). ${ }^{3}$ Both AOTF and LCTF crystals are polarization-dependent and have a low light-transmission rate ( $25 \%$ AOTF, $5 \%$ to $35 \%$ LCTF). They have an out-of-band blocking power that is less than 3 to 4 OD in the case of AOTF and 2 OD in that of LCTF. TFTF applications in hyperspectral imaging are just beginning; they show a high transmission rate $(90 \%)$, a full visible spectrum acquisition, a bandwidth of 13 to $16 \mathrm{~nm}$, a high out-of-band rejection (5 OD), but they have a slow acquisition speed. ${ }^{3}$ Several of these systems have been used in live cell microscopy, for instance, in Förster resonance energy transfer (FRET), ${ }^{6}$ in discriminating between autofluorescence and GFP fluorescence ${ }^{7}$ and in bulk quantum dot (QDs) tracking in non-neuronal cells. ${ }^{8}$ However, no hyperspectral system has been exploited for single-molecule imaging in live neurons.

Here, we present an application for a hyperspectral tunable filter originally designed for astrophysics. ${ }^{9}$ It is applied to live neuron optical imaging and allows single-molecule detection and tracking. The hyperspectral microscopy system that we (Photon etc.) developed uses a resonant Bragg tunable filter (BTF) imaging spectrometer mounted in front of an EMCCD camera. The BTF shows a high light-transmission rate, broad wavelength tuning range, and a fast spectrum acquisition rate. Furthermore, spectral properties of the tunable filters are almost identical for both $s$ and $p$ polarizations of light. 
We and others have used QDs as labels for tagging membrane receptors in cultured neurons and tracked their displacement during synaptic signaling and remodeling. ${ }^{10,11}$ With their narrow emission spectra, bright fluorescence, and robust photostability, QDs are ideally suited for multispectral imaging of single proteins. We tested the hyperspectral imaging system for single-molecule tracking of QD-tagged membrane receptors in live, cultured hippocampal neurons. We report the simultaneous tracking of five proteins in the same neuron: a GFP-tagged synaptic marker (GFP-CaMKII) and four glutamate receptors or associated proteins (GluA1, GluA2, mGluR5, and Stargazin) tagged with QDs of different emission wavelengths. This approach can test the impact of various compounds on the spatial dynamics of several proteins or receptors simultaneously. As a proof of concept, we tracked multiple subtypes of individual receptors in the membrane of living neurons in the presence or absence of glutamate. Our results establish that our system can be used to assess the mobility of multiple subtypes of receptors in neurons treated with different reagents. This should provide a useful tool for drug screening applications.

\section{Methods}

\subsection{Primary Neuronal Cultures, Transfection}

Neonatal rats aged from 1 to 3 days (P1 to P3 rats) were sacrificed by decapitation, using procedures recommended by the animal care committee of Universite Laval. Their hippocampi were then dissected. Hippocampal cells were dissociated enzymatically and mechanically (trituration through a Pasteur pipette). A papain solution $(12 \mathrm{U} / \mathrm{ml}$; Worthington, Freehold, New Jersey) containing $\mathrm{Ca}^{2+} / \mathrm{Mg}^{2+}$-free $\mathrm{HBSS}, 0.42 \mathrm{mg} / \mathrm{ml}$ cysteine (Sigma), $250 \mathrm{U} / \mathrm{ml}$ DNase 1 (type IV; Sigma), $25 \mathrm{mM}$ NaHCO3, penicillin $(50 \mathrm{U} / \mathrm{ml}) /$ streptomycin $(50 \mu \mathrm{g} / \mathrm{ml})$, $1 \mathrm{mM}$ sodium pyruvate, and $1 \mathrm{mg} / \mathrm{ml}$ glucose was used. After dissociation, cells were washed and centrifuged in a Neurobasal medium containing BSA $(2 \mathrm{mg} / \mathrm{ml}$ and $20 \mathrm{mg} / \mathrm{ml}$, respectively), Pen/Strep, glucose, pyruvate, and DNase1 (as above). They were plated on poly-D-lysine-coated Aclar coverslips at high density $\left(1 \times 10^{6}\right.$ cells $/ 18 \mathrm{~mm}$ coverslip). Growth media ( $2 \mathrm{ml} /$ well) consisted of a mixture of Neurobasal medium and B27, supplemented with penicillin/streptomycin $(50 \mathrm{U} / \mathrm{ml}$; $50 \mu \mathrm{g} / \mathrm{ml}$ ), and $0.5 \mathrm{mM}$ Glutamax (Thermo Fisher Scientific, Waltham, Massachusetts). Cytosine $\beta$-D-arabinofuranoside (Ara-C) $(5 \mu \mathrm{M}$; Sigma, St. Louis, Missouri) was added $24 \mathrm{~h}$ after plating to reduce the number of non-neuronal cells. After 4 days in vitro (DIV), half of the growth medium was replaced twice a week by a medium containing no Ara-C.

After 12 to $14 \mathrm{DIV}$, neurons were cotransfected with Lipofectamine 2000 and GFP-CaMKII WT, FLAG-GluA1, myc-mGluA5, AP-GluA2, BirA, and HA-Stargazin DNAs. DNA and lipofectamine were applied for $3 \mathrm{~h}$ and coverslips were then transferred to fresh growth media. Neurons were imaged 18 to $24 \mathrm{~h}$ after transfection.

\subsection{Quantum Dots, Antibodies, Receptor Labeling, Imaging Solution, and Drug Application}

QDs $605 \mathrm{~nm}$ goat $\mathrm{F}\left(\mathrm{ab}^{\prime}\right) 2$ anti-rat, QD655 nm streptavidin, QD705 nm anti-mouse, and QD805 nm anti-rabbit conjugates $(\mathrm{H}+\mathrm{L})$ highly cross-adsorbed were bought from Thermo Fisher Scientific. These different QDs $(0.1 \mu \mathrm{M})$ were precoated by incubation with 0.1 to $0.2 \mu \mathrm{g}$ of primary antibody in $10 \mu \mathrm{l}$
PBS for 30 min [either the Roche rat anti-HA (clone 310F), Life Technologies (Carlsbad, California) mouse anti-c-Myc or Sigma rabbit anti-FLAG]. Dilution $(0.1 \mathrm{mM})$ of QD655-streptavidin for biotinylated-GluA2 was made in PBS. Unspecific binding was blocked by adding casein at $1 \times$ (Vector Labs, Burlingame, California) to the precoated QDs $15 \mathrm{~min}$ before use. To visualize synapses while tracking the diffusion of four membrane receptors in live hippocampal neurons, we co-transfected GFP-CaMKII with the tagged receptors HA-Stargazin, AP-GluA2, BirA, Myc-mGluR5, and FLAG-GluA1. The four stock solutions of precoated QDs were combined in a single "QD-PBS" solution. This solution was then added (final dilution of 0.02 to $0.1 \mathrm{nM}$ ) for 20 min to neurons in culture medium at $37{ }^{\circ} \mathrm{C}$. This incubation was followed by two washing steps of $30 \mathrm{~s}$ each in a standard imaging solution, consisting of HBSS (Thermo Fisher Scientific) containing (in mM): 10 HEPES, 2.0 glucose, $1.2 \mathrm{CaCl}_{2}$, and $1.0 \mathrm{MgCl}_{2}$. We then fixed the neurons with ethanol (Fig. 5) and mounted the coverslips on slides using Qmount mounting media (Thermo Fisher Scientific). For live imaging, the QD-PBS solution was incubated with or without glutamate $(100 \mu \mathrm{M})$ for $20 \mathrm{~min}$ in the culture medium (Fig. 8).

\subsection{Receptor Tracking and Analysis}

The tracking of single QDs was performed with a homemade software based on MATLAB ${ }^{\circledR}$ (Mathworks Inc., Natick, Massachusetts). Single QDs were identified by their blinking fluorescent emission and their diffraction limited signals. Because of the QD blinking properties, trajectories of QD-tagged receptors could not be tracked continuously. Subtrajectories of a single receptor were reconnected when its positions before and after the dark period were compatible with borders set for maximal position changes between consecutive frames and blinking rates. These values were determined empirically: 5 pixels $(1.3 \mu \mathrm{m})$ for maximal position change between two frames and maximal dark periods of 5 frames (15 s). Mean square displacement (MSD) curves were calculated for reconnected trajectories of at least 10 frames. Diffusion coefficients were calculated by a linear fit of the first four points of the MSD plots versus time.

\subsection{Statistics}

Values are expressed as mean \pm SEM or median $\pm 5 \% / 95 \%$ interval. Statistical significances were calculated with the MATLAB ${ }^{\circledR}$ Statistical Toolbox. Non-Gaussian distributed data sets were tested with the Wilcoxon test for paired observations. Indications of significance correspond to $p$ values $<0.05(*)$, $p<0.005(* *)$, and $p<0.0005(* * *)$.

\section{Results}

\subsection{Development of a Hyperspectral Microscopy System for Multiplex Imaging}

The image acquisition system consisted of an Olympus IX-71 inverted microscope (Fig. 1), equipped with a $60 \times$ oil objective (Plan Apo N, N.A. 1.4, Olympus, Tokyo, Japan), Xenon-arc lamp (Lambda LS, Sutter Instrument, Novato, California), filter cube (ex 447/60 nm; dichroic LP495 nm, Semrock), BTF (Photon Etc.), and an EMCCD camera (HNü512, Nüvü Cameras, Montreal, Canada). All hardware controls and acquisition 

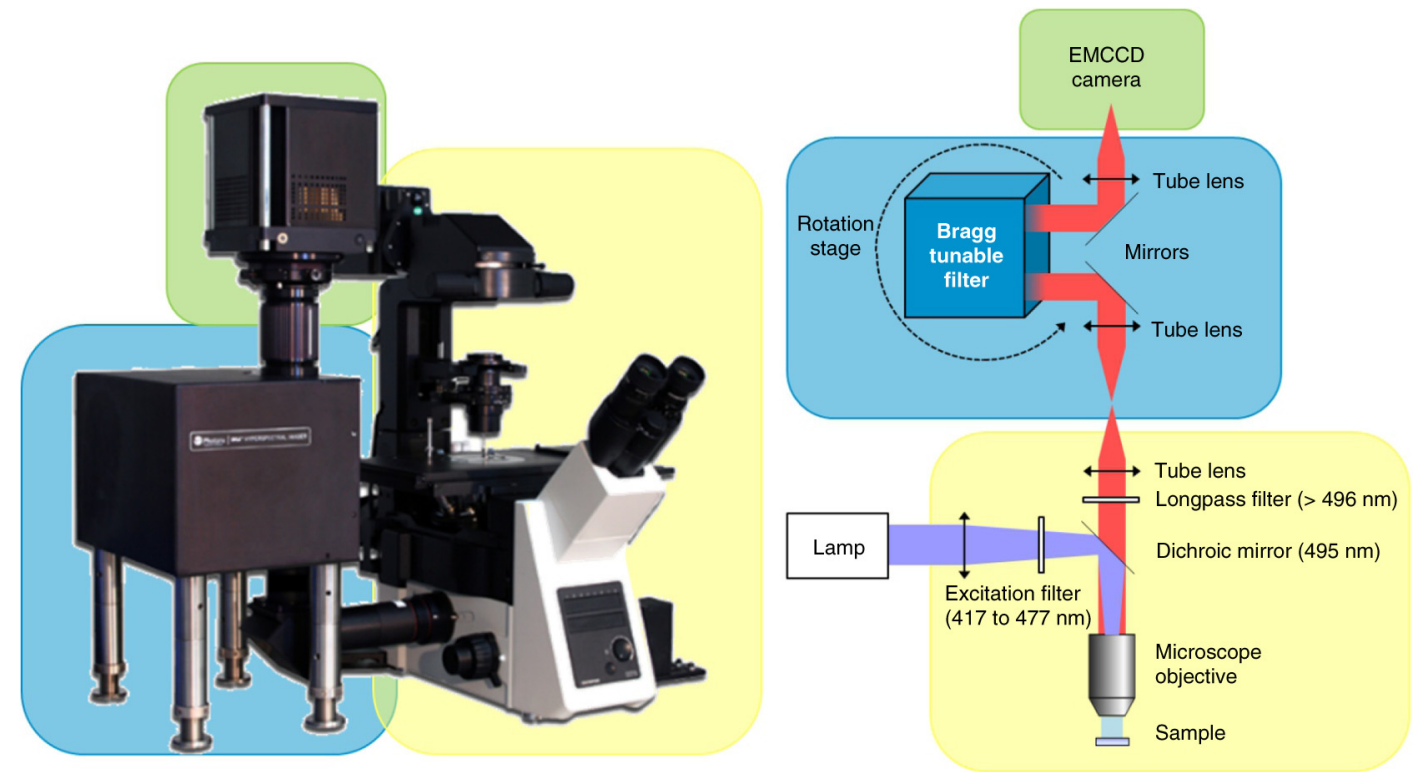

Fig. 1 Scheme of the multiplex hyperspectral imaging platform with the main components: microscope (yellow background), hyperspectral filter (blue background), and camera (green background).

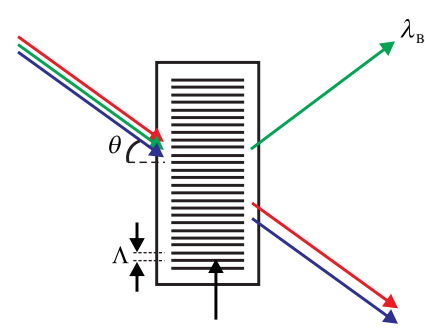

Plane of index (top view)

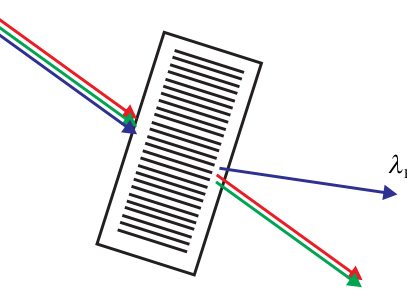

$\lambda_{\mathrm{B}}=2 n \Lambda \sin (\theta)$ (a)

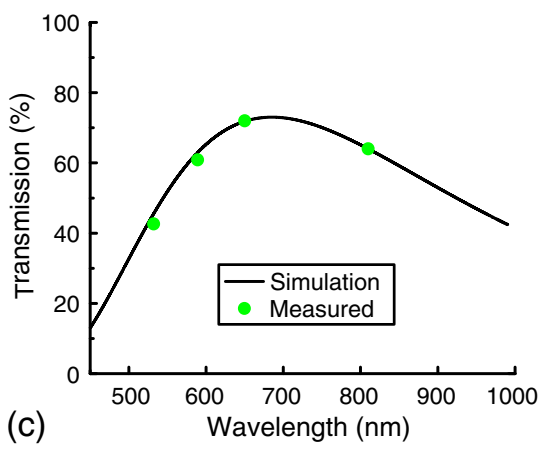

Fig. 2 Filtering scheme for transmission BTF and current design of a transmission imaging BTF using a volume hologram. (a) BTF wavelength selection. Changing the incident angle on the BTF changes the selected output wavelength (b) light path inside the hyperspectral filter. (c) BTF system transmission measured values for four lasers 532, 589, 650, and $810 \mathrm{~nm}$ (dots) and volume Bragg grating simulation results (line). (d) Out of band rejection of the Bragg filter for a 700-nm laser line. (e) Out of band rejection for the entire BTF microscope system measured with 532 and $589 \mathrm{~nm}$ lasers. 
parameters were integrated in the Photon Etc. proprietary software "Physpec."

The BTF optical core is a Bragg volume hologram in which the refraction index varies periodically. ${ }^{12}$ When a collimated polychromatic beam reaches the BTF, only a particular narrow bandwidth satisfies the Bragg condition and constructively interferes with the refractive index modulation, while all other wavelengths are not diffracted [Fig. 2(a)]. The BTF was positioned on one of the camera port of the microscope. The full image in the field of view is sent to the BTF through two tube lenses that are used as a relay in order to image the pupil on the BTF.

The spectrum acquisition of the polychromatic light emitted by the sample follows these steps. First, light crosses the transmission Bragg volume hologram that selects a specified wavelength; then a mirror corner cube is used to redirect the light to a second region of the grating [Fig. 2(b)]. This second filtering step narrows down the bandwidth, compensates for the dispersion, and delivers an out-going beam in a direction parallel to the incoming one. The corner cube and the volume hologram are positioned on a rotation stage. By modifying the angle of the stage, the incident angle of the incoming light on the Bragg volume hologram is modified, thereby selecting another wavelength. The resonant wavelength can be tuned over hundreds of nanometers. The size of the gratings was designed to accommodate the pupil size of commercial microscope objectives in a way that the surface of the collimated beam does not exceed the grating. Thus, objectives with numerical aperture ranging from 0.1 to 1.4 produce pupil size below the Bragg gratings clear aperture $(>12 \mathrm{~mm})$. Then, the NA has no effect on the bandwidth, out-of band rejection, and transmission.

One mode of spectra acquisition is done in a step-by-step scanning mode. The filter is positioned at a determined wavelength and stands still while the EMCCD camera is acquiring an image. Once the image is acquired, the rotation stage is repositioned at the next determined wavelength. A new image is then acquired. This process is repeated to acquire filtered images covering the desired wavelength range. This mode renders precise spectra but is fairly slow for full spectra acquisition. This is why we developed a fast spectrum acquisition called continuous acquisition. This mode follows a continuous motion by streaming the camera while constantly changing the angle of the rotation stage. During this scanning process, the camera produces a TTL synchronization signal in order to reconstruct the spectral information of the images. This mode has a higher acquisition speed and produces an increase in the spectral bandwidth of the data collected, combining the intrinsic filter bandwidth and the acquisition interval. To quantify this effect, we measured the $703.24 \mathrm{~nm}$ peak FWHM of a Ne calibration lamp, and found

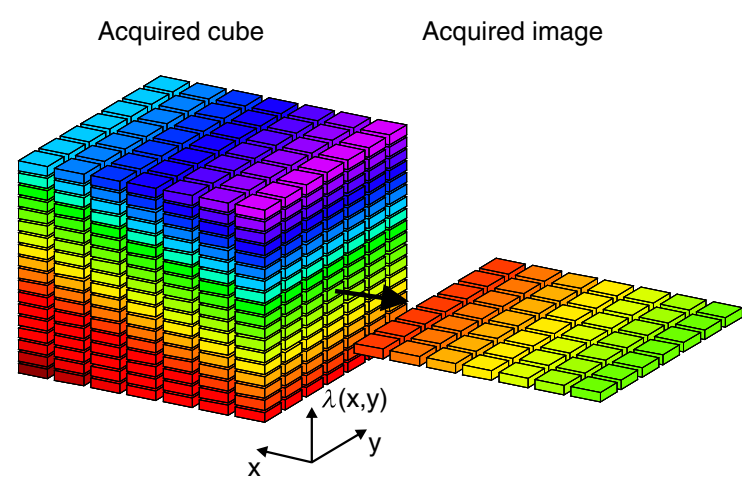

(a)

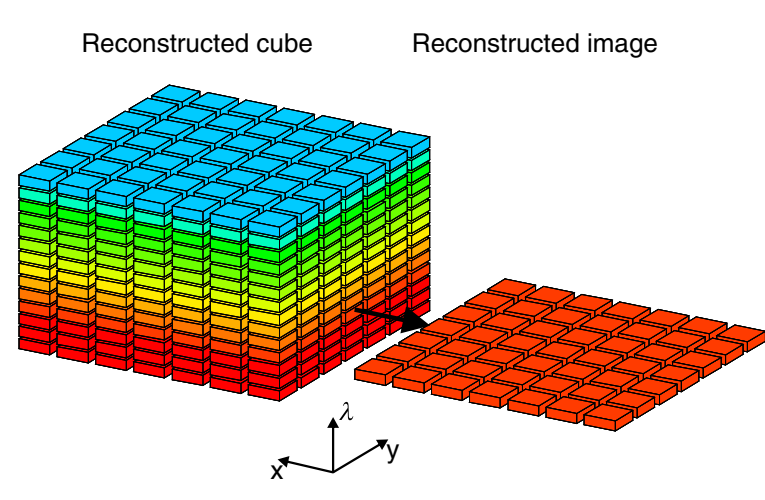

(c)

(b)
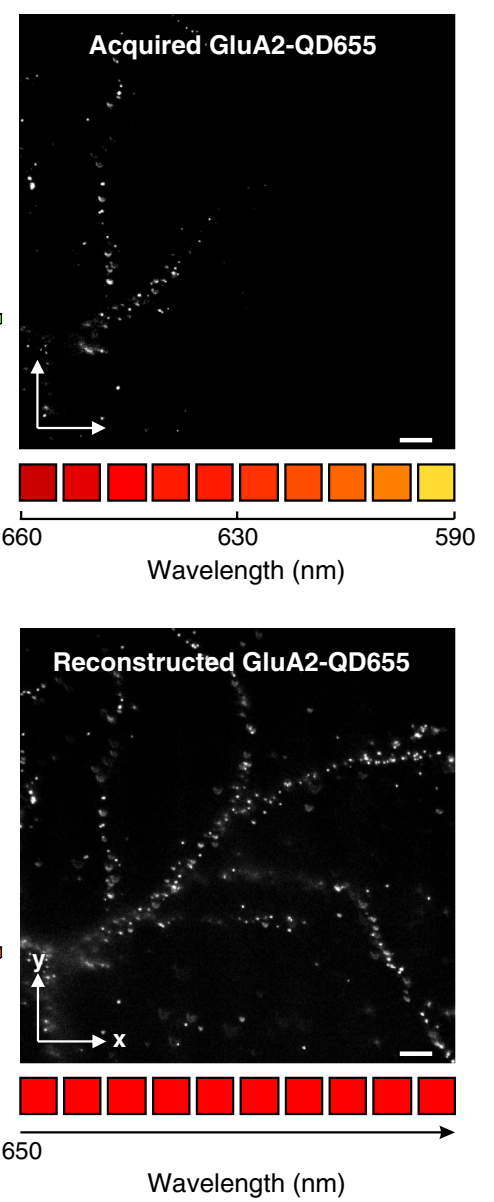

Fig. 3 BTF hyperspectral acquisition and image reconstruction. (a) Acquired images with a gradient in wavelength in the dimension parallel to the dispersion axis and (c) reconstructed monochromatic images. (b and d) Acquisition and reconstruction images of a neuron overexpressing membrane AP-GluA2 tagged with streptavidin $655 \mathrm{~nm}$ QDs. The narrow emission spectra of the QD 655 spreads over a few pixels of the camera (b) and sweeping the peak wavelength on the whole camera chip allows the reconstruction of the monochromatic image (d). Scale bars $10 \mu \mathrm{m}$. 
that in the step-by-step mode the FWHM was $1.8 \mathrm{~nm}$, while in the continuous mode it was $8 \mathrm{~nm}$. All experiments were done in the continuous acquisition mode.

The BTF microscope also allows to choose the spectral step acquisition, without changing the optical properties of the BTF. The user can thus select the sampling frequency. For example, we used a 2-nm step increment for high-resolution spectra of single QDs. However, for high frame rates, we chose a $10-n m$ step increment. Finally, when using one or two fluorescent probes, it is possible to select a part of the spectrum related to the probe emission spectra without scanning the whole spectrum.

\subsection{Bragg Tunable Filter Microscope Characterization and Performance}

To evaluate the level of transmission, we used four lasers (532, 589,650 , and $810 \mathrm{~nm}$ ) that were injected in a single-mode fiber (9.8 $\mu \mathrm{m}$ core diameter). The optical fiber output was placed in the center of the field of view and in the focal plane of the microscope with a $10 \times$ objective. As the core diameter is much smaller than the field of view of the objective, this simulates a punctual source, leading to a collimated beam on the BTF. The incident power on the BTF and the output power of the filtered beam after passing through the BTF were measured with a power meter and a photodiode (Thorlabs PM100D and S121C, Thorlabs Inc., Newton, New Jersey). The ratio of these two values gives the efficiency of the BTF at the laser wavelengths [Fig. 2(c)]. Also, the diffraction efficiency curve of the volume Bragg grating has been calculated using the couple-mode theory ${ }^{12}$ based on design parameters, such as the thickness of the grating, the period and the amplitude of the index modulation recorded in the photo-thermorefractive glass. In this calculation, Fresnel losses have been taken into account.

The optical density of the volume Bragg grating has been characterized on a Photon etc. tunable filter using a collimated supercontinuum source (Fianium's WL SC480-10, Southampton, UK). The transmission spectrum shown in Fig. 2(d) exhibits an optical density greater than 6 . However, the optical density measured with 532 and $589 \mathrm{~nm}$ laser lines in the actual system is limited to OD3 because of the background stray light [Fig. 2(e)]. No effort was made to minimize this background since the QD optical signal was already sufficient to be discriminated and tracked. Further optical confinement of the optical path can be added for the detection of a weaker signal.

On the BTF, the wavelength tuning range was designed to vary from 500 to $900 \mathrm{~nm}$ with a bandwidth of $2.2 \mathrm{~nm}$. To evaluate the full spectrum acquisition time, we used the same parameters as those used by Favreau et al. ${ }^{3}$ Hyperspectral image stacks of fluorescence emission were acquired over a wavelength range of 470 to $700 \mathrm{~nm}$ in 5-nm increments, 500-ms acquisition time for each frame. These authors reported a total spectral image acquisition time with the AOTF system of $\sim 30 \mathrm{~s}$, and TFTF was $\sim 126 \mathrm{~s}$. In our case, using the same parameters for the $\mathrm{BTF}$, the acquisition time was $\sim 34 \mathrm{~s}$.

For a change in wavelength of $3 \mathrm{~nm}$ or less, the tuning speed is $60 \mathrm{~ms}$. The maximum scanning speed in continuous mode for a spectra of 500 to $850 \mathrm{~nm}$ is less than $1 \mathrm{~s}$. However, in order to collect enough photons for live-cell experiments using QDs, we set the exposure time at $50 \mathrm{~ms}$ leading to a 3.4-s frame rate for a 500 to $850 \mathrm{~nm}$ spectrum with a $10-\mathrm{nm}$ step size.

\subsection{Hyperspectral Bragg Tunable Filter Acquisition}

The original images taken during spectrum acquisition are not monochromatic. The angular selectivity of the grating leads to a gradient in wavelength across the camera field of view, in the dimension parallel to the dispersion axis [Fig. 3(a)]. The gradient wavelength " $\Delta \lambda$ " in images is defined as

$\Delta \lambda=2 \Lambda \cdot \frac{\Delta x}{f} \cdot \sqrt{1-\left(\frac{\lambda}{2 \Lambda}\right)^{2}}$,

where " $\Delta x$ " is the number of pixels (512) multiplied by the pixel size $(16 \mathrm{~mm}), " \Lambda$ " is the grating period $(960 \mathrm{~nm}), " \lambda$ " is the

(a)
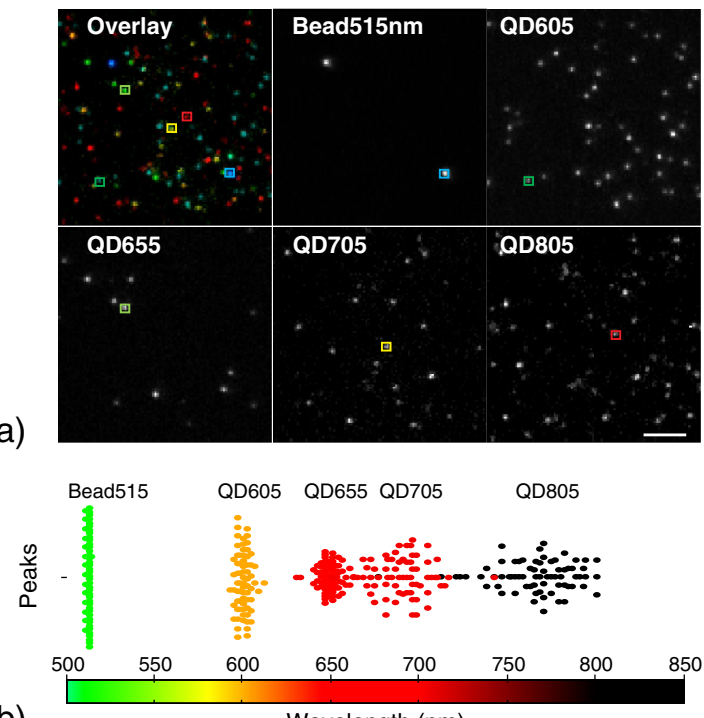

(b)

Wavelength $(\mathrm{nm})$
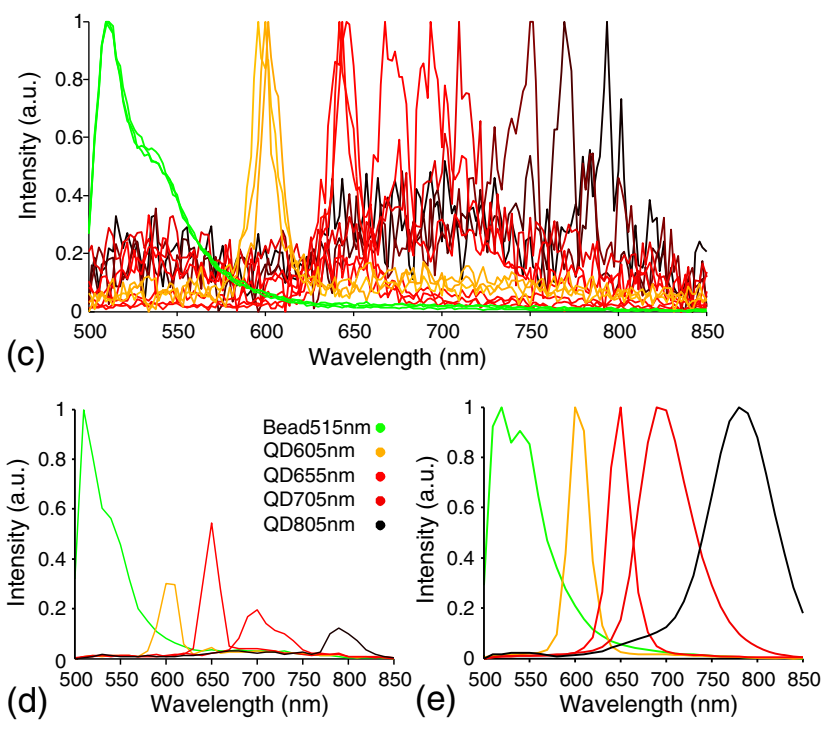

Fig. 4 Hyperspectral measurement of single QD emission spectra. (a) Images of dispersed QDs on a glass cover slip. Single QDs were identified based on their intensity, blinking, and emission spectrum. Scale bar $5 \mu \mathrm{m}$. (c) Maximum peak intensity of different types of single beads and QDs: Bead515 nm; QD605 nm; QD655 nm; QD705 nm; QD805 nm; $n=70$ for all wavelength. (c) Representative spectra of 15 single beads and QDs (three for each color) acquired with a 2-nm step size. (d) Spectra of single QDs taken with a 10-nm step. (e) Spectra average from more than 1000 QDs dispersed on glass (10 nm step). 
wavelength, and " $f$ " is the output focal length of the instrument (200 mm). Applying these figures to Eq. (1) leads to a gradient wavelength of roughly $70 \mathrm{~nm}$ along one of the axis of the camera chip for an emission wavelength centered at $650 \mathrm{~nm}$. Is it important to note that the gradient would differ for a camera with pixels different in size and number.

To demonstrate the hyperspectral cube acquisition, we transfected neurons with AP-GluA2 and biotin ligase (BirA). Once AP has been biotinylated by BirA, it allows the expression of biotinylated-GluA2 on the membrane. ${ }^{13}$ Labeling was performed using $0.1 \mathrm{nM}$ of streptavidin-conjugated QDs emitting at $655 \mathrm{~nm}$ (SA-QD655). The narrow emission spectra (FWHM $28 \mathrm{~nm}$ ) of QD655 spread to a few pixels on the acquisition image [Fig. 3(b)]. To get a monochromatic image, a broader spectral acquisition was scanned allowing the reconstruction of the monochromatic image at the selected wavelength [Fig. 3(c)].
In fact, the acquisition automatically expands the raw spectral range in order to have enough data to build all monochromatic images requested by the user. The software computes the monochromatic images through a cubic interpolation between the pixels with the same position acquired through the four closest raw wavelengths (cubic spline on the 2 wavelengths lower and higher than the wavelength of interest). The interpolation can produce spectral artifacts but is limited when the Nyquist criterion is achieved. Therefore, data acquisition must be achieved with a wavelength step at least half of the narrowest spectral FWHM present in the signal. Acquired pixel lines that exceed the user's selected wavelength are not used in the reconstruction of the monochromatic image. The reconstruction process involves no background subtraction or correction for wavelength-dependent attenuation. The reconstructed image of the neuron labeled with SA-QD655 is shown in Fig. 3(d). Reconstruction of the

(a)

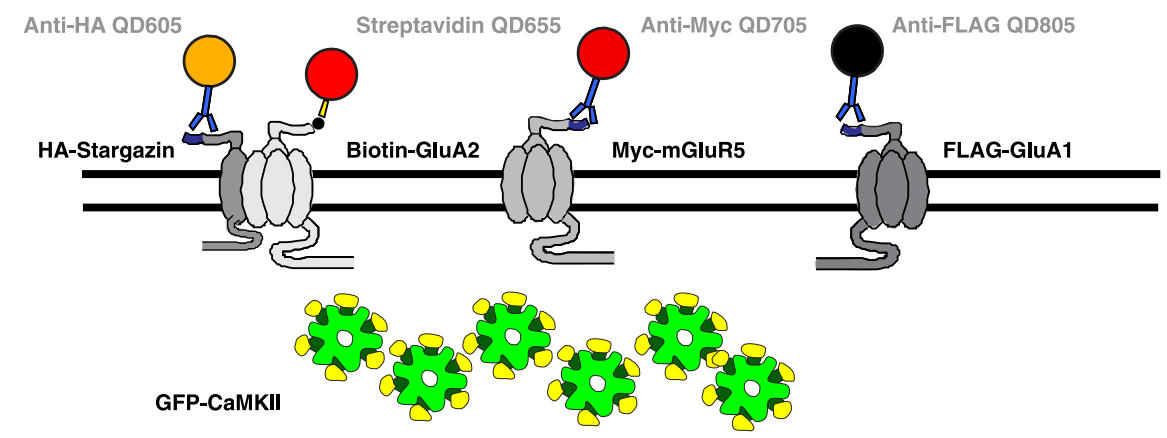

(b)

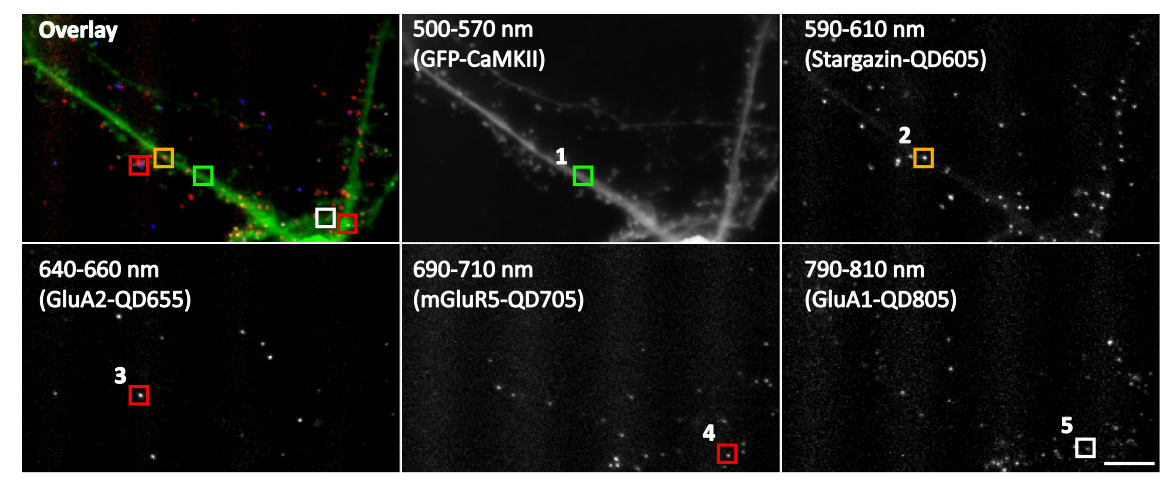

(c)

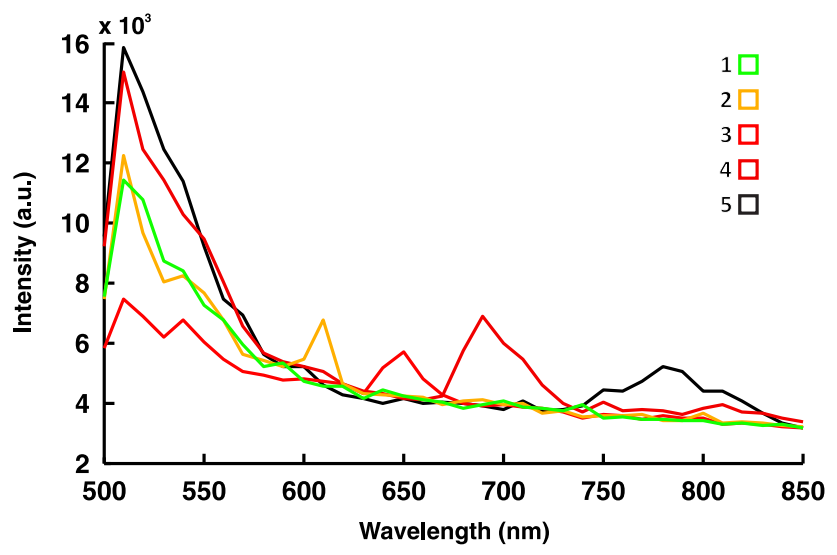

Fig. 5 Labeling and detection of five markers on neurons. (a) Labeling scheme for tracking four membrane receptors and one synaptic marker. (b) Hyperspectral detection of four membrane receptors tagged with QDs on a GFP-CaMKII expressing neuron; scale bar $10 \mu \mathrm{m}$. (c) Intensity spectra of single QDs and CaMKII [colored and numbered squares from (b)]. Note that the measured spectra in (c) are mixed spectra from QDs and GFP-CaMKII. The neuron in this image was fixed before imaging. 
images was done online in "Physpec," the Photon Etc. acquisition systems.

\subsection{Single-Molecule Hyperspectral Detection of Five Fluorescence Markers on Glass}

In order to assess the capability of the imaging system to distinguish five fluorescents markers, we dispersed on a glass cover slip a mixture of $515 \mathrm{~nm}$ beads $(0.1 \mu \mathrm{m}$, Thermo Fisher Scientific) and QDs of four different diameters: 605, 655, 705, or $805 \mathrm{~nm}$. Because the purification of GFP is tedious, we selected $515 \mathrm{~nm}$ beads, as their emission spectra are similar to that of GFP. To increase the spectral resolution, we selected a 2-nm step for spectra acquisition. Single beads and QDs were identified on the basis of their intensities [Fig. 4(a)]; the spectrum and fluorescence peak values were extracted. We noticed that the peak value distribution of the emission was broader for QD $700 \mathrm{~nm}$ and QD $805 \mathrm{~nm}$ than for the other QDs [Fig. 4(b)]. Interestingly, the full range of single QD emission spectra for QD605, QD655, and QD705 showed a typical Gaussian shape, whereas the emission spectra of QD805 displayed a maximum near $800 \mathrm{~nm}$ and a bimodal fluorescence curve that contained a weaker fluorescence signal from 625 to $750 \mathrm{~nm}$ [Fig. 4(c)]. Note that QD blinking while acquiring spectrum may alter the shape of the single QD emission spectra.

Then, we tested whether the hyperspectral system can discriminate five different markers. To enable single-molecule tracking of the different labeled receptors, we used a larger step size $(10 \mathrm{~nm})$, thus ensuring an increased acquisition rate. Figure 4(d) shows a single QD spectrum taken with a 10-nm (a)
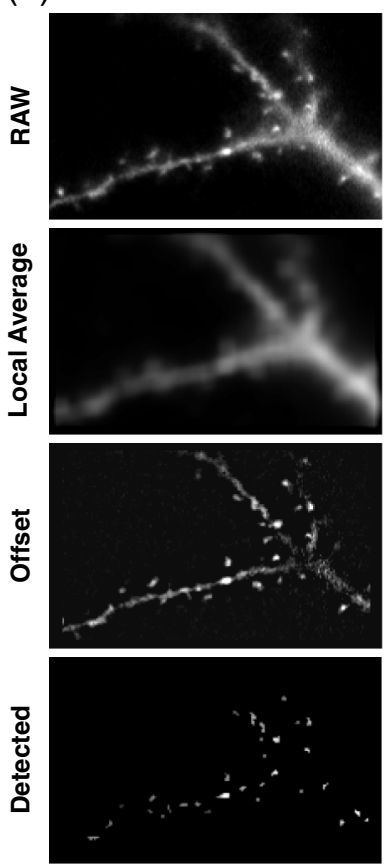

(b)
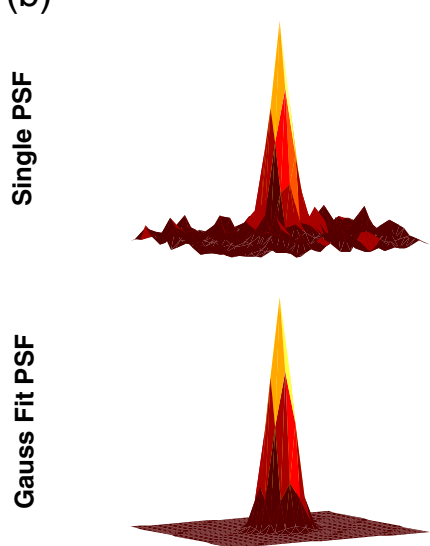

$\mathrm{N}=128 \pm 30$ photons $\mathrm{r}=115 \pm 40 \mathrm{~nm}$
Stargazin QD605
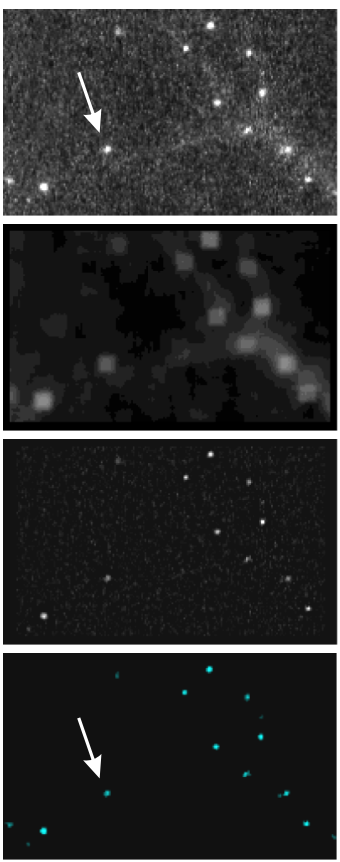

QD655
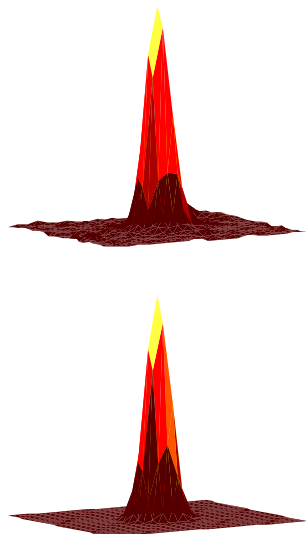

$\mathrm{N}=144 \pm 44$ photons $\mathrm{r}=115 \pm 55 \mathrm{~nm}$
GluA2 QD655
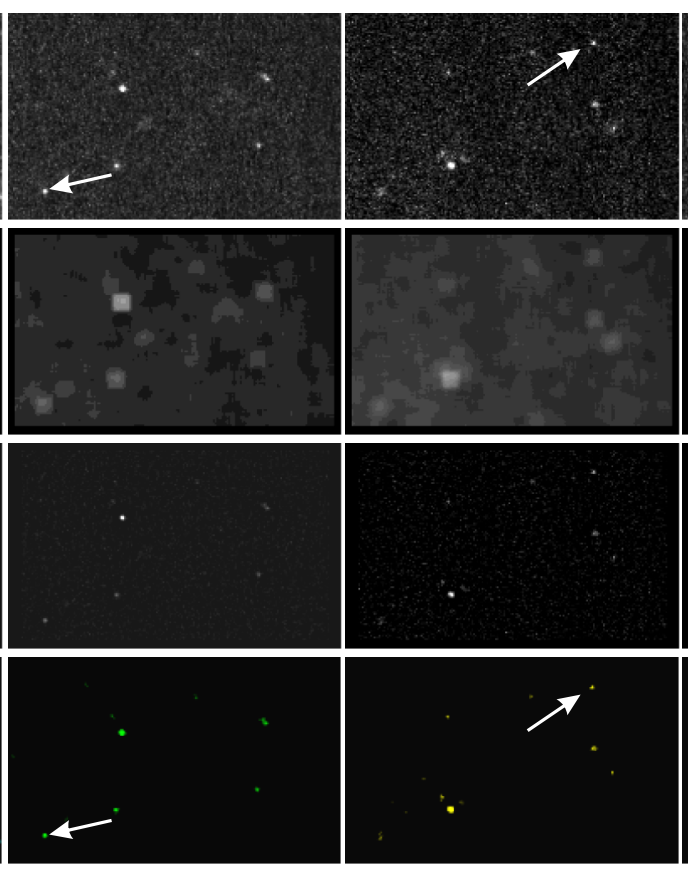

QD705
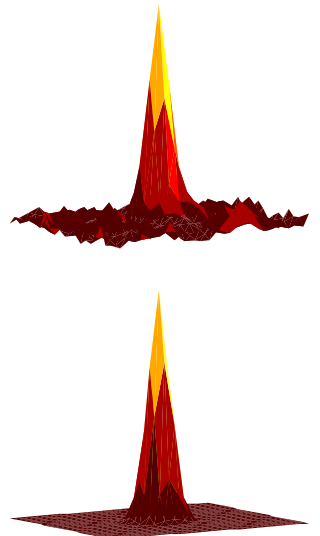

$\mathrm{N}=136 \pm 15$ photons $r=96 \pm 54 \mathrm{~nm}$
GluA1 QD805
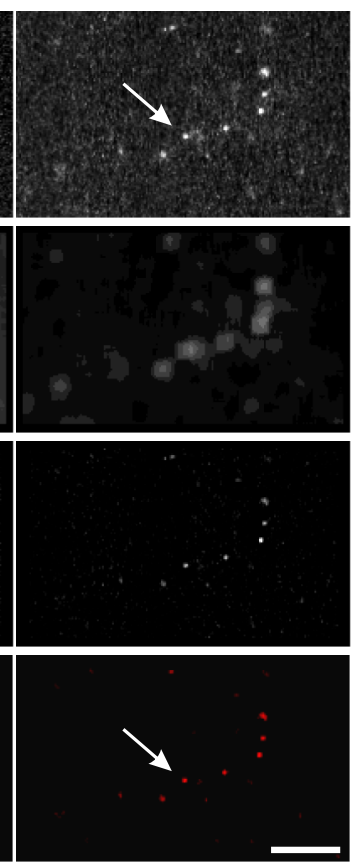

QD805
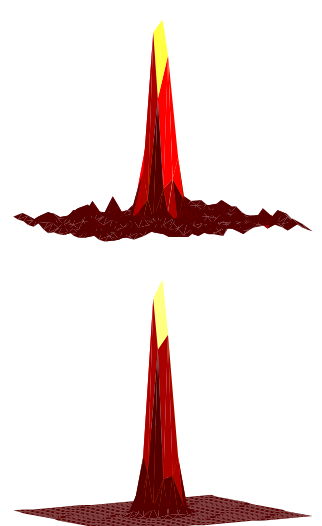

$\mathrm{N}=103 \pm 58$ photons $r=103 \pm 58 \mathrm{~nm}$

Fig. 6 Detection and subpixel localization of single QDs with a hyperspectral microscope. (a) The detection algorithm uses local averaging and morphological analysis to extract single QDs. Scale bar $10 \mu \mathrm{m}$.

(b) Examples of single QD PSFs and Gaussian fittings. Accuracy on localization for each QD $(n=2199$

to 5125$)$ is shown in mean \pm SEM. Averaged background for all channels was $2 \pm 1$ photons. 
step size; its "saw-tooth-like" appearance is due to the low sample rate $(10 \mathrm{~nm})$ and QD blinking. Figure 4(e) shows an average of spectra $(>1000)$ for each population of individual QDs, which are in very good accordance with the expected values published by the manufacturer (Thermo Fisher Scientific). This demonstrates the capability of the hyperspectral system to detect and discriminate five fluorescent markers of different emission wavelength in the same sample.

\subsection{Hyperspectral Detection of Five Fluorescence Markers on Fixed Cells}

We then tested the feasibility of measuring the spectra of GFP, QD605, QD655, QD705, and QD805 on the same neuron. We transfected neurons with GFP-CaMKII and HA-Stargazin, APGluA2, BirA, Myc-mGluR5, and FLAG-GluA1. Twenty-four hours later, the neurons were incubated for $20 \mathrm{~min}$ with QDs anti-HA-605, Streptavidin-655, anti-Myc-705, and antiFLAG-800 nm [Fig. 5(a)], fixed and mounted on slides with Qmount mounting media (Thermo Fisher Scientific). We imaged the cells on the hyperspectral microscope with a 10$\mathrm{nm}$ step size from 500 to $850 \mathrm{~nm}$ [Fig. 5(b)]. Single QDs were identified based on their size and peak emission; we then extracted the hyperspectral values of single QDs for each wavelength. Figure 5(c) shows the fluorescent signal spectra of the four QDs and GFP-CaMKII, demonstrating that each QD can be discriminated based on its emission spectrum on a cell that is expressing GFP.

\subsection{Live Cell Detection, Gauss Fit, Localization Accuracy, and Tracking Multiple Quantum Dots}

We performed imaging with the hyperspectral microscope in live neurons, previously cotransfected with GFP-CaMKII, HAStargazin, AP-GluA2, BirA, Myc-mGluR5, and FLAG-GluA1 and labeled with the corresponding tagged QDs. To generate movies, we acquired 100 spectra of 500 to $850 \mathrm{~nm}$ and selected five wavelength windows that allowed us to reconstruct movies for each color (500 to $570 \mathrm{~nm}, 590$ to $610 \mathrm{~nm}, 640$ to $660 \mathrm{~nm}$, 690 to $710 \mathrm{~nm}$, and 790 to $810 \mathrm{~nm}$ ).

For detection of a single QD, we used an adaptive threshold algorithm; first, we obtained a local average image using a convolution of a square of " $N$ " pixels, the sum of which is equal to 1 , with the original image to obtain an image that represents local average for each pixel (Fig. 6). Then, the original image was subtracted to the local average image obtaining an offset image. Brighter spots were identified as pixels having intensities higher than the mean plus 0.5 standard deviation of the offset image. Finally, the adaptive algorithm was coupled to a morphological analysis that scores objects having a size between 3 and 100 pixels. The centroid of each aggregate was used as the initial value in the Gaussian regression [Fig. 6(b)]. This algorithm of adaptive threshold coupled with morphological analysis was also used for the detection of synapses using fluorescent synaptic markers (GFP-CaMKII).

Before locating single QDs, the mean value of the camera noise (determined as the average of signals from 10 images taken with the shutter closed under the same conditions of gain and exposure time) was subtracted from each image of the QD movies. Single QDs were localized by fitting a Gaussian function to a $9 \times 9$ pixel array surrounding the peak. The number of photons was calculated first by multiplying the count values (a.d.u.) of the array with the manufacturer conversion factor
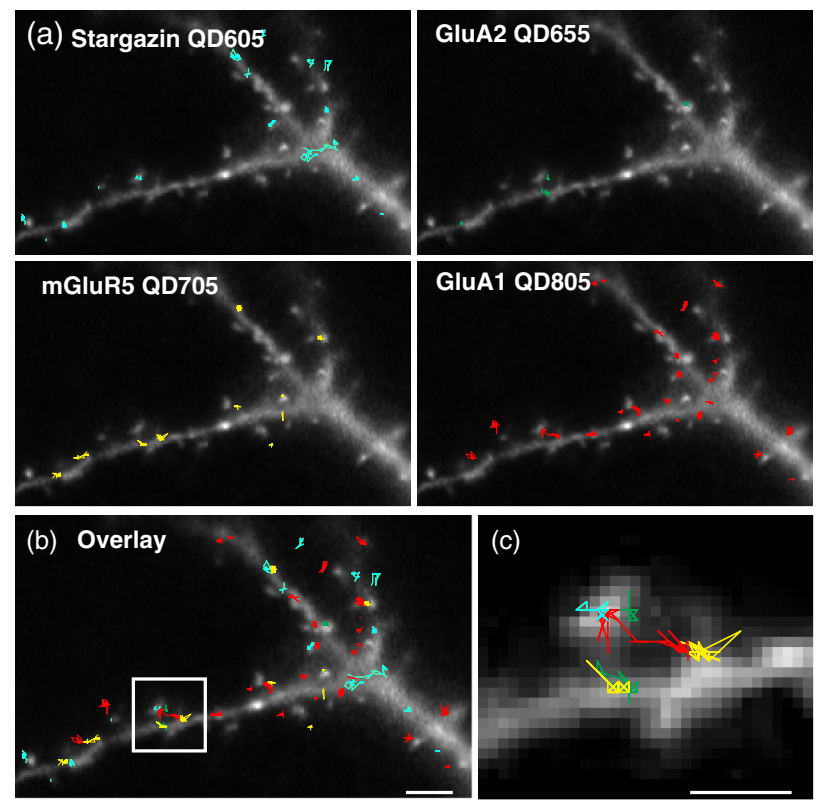

Fig. 7 Color-coded tracking of four membrane receptors on a live neuron. (a) Stargazin-QD605 trajectories are shown in cyan, GluA2-QD655 in green, mGluR5-QD705 in yellow, and GluA1QD805 in red. (b) Overlay of the four receptor tracks on the GFPCaMKII signal; scale bar $5 \mu \mathrm{m}$. (c) Tracking of the four receptor subtypes in a single spine region. Scale bar $5 \mu \mathrm{m}$.

(photons/a.d.u.), then by summing the single QD arrays. Error on localization was calculated with ${ }^{14}$

$\Delta x y=\sqrt{\frac{s^{2}}{N}+\frac{a^{2} / 12}{N}+\frac{8 \pi s^{4} b^{2}}{a^{2} N^{2}}}$,

where $s$ is the full width at half maximum of the fitted PSF, $a$ is the pixel size $(0.267 \mu \mathrm{m})$, and $b$ is the background (mean of the perimeter pixels of the single QD array). Number of photons $N$ of the PSF was converted from the camera values with the conversion factor provided by the manufacturer (Nüvü Camera). Accuracy on localization and background values for single PSF were calculated for each QD wavelength.

The particle detection algorithm described above generates a set of positions for each image sequence; by connecting these positions, we can obtain trajectories. Single QD with localization accuracy better than $250 \mathrm{~nm}$ were entered into the tracking algorithm and locations were assembled into tracks using a freely available algorithm developed by Crocker (Fig. 7). We obtained cases where all five labeled proteins and receptors colocalized in the same synapse structure and adjacent dendrite (Fig. 7). To our knowledge, this is the first demonstration of simultaneous tracking of four receptor subtypes in living neurons.

\subsection{Tracking Multireceptor Subtypes on Live Neurons, Following Pharmacological Treatment}

To test the capability of the hyperspectral system to be used as a platform for drug discovery on live neurons, we applied glutamate, which is known to impact on receptor mobility, ${ }^{11}$ onto the neurons, prior to imaging. We measured the diffusion coefficient of each receptor and compared them to control condition. The results indicate that Stargazin mobility was strongly reduced, 
(a)

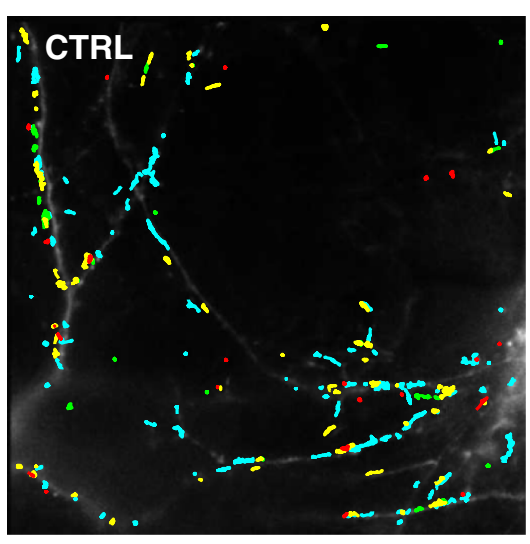

(b)

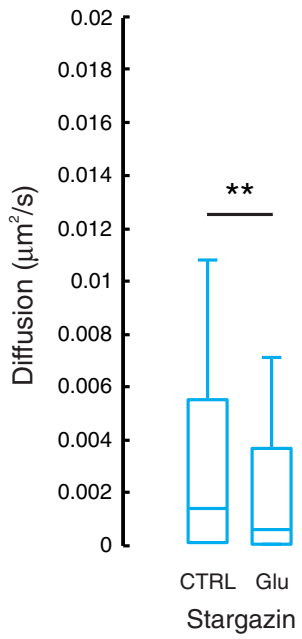

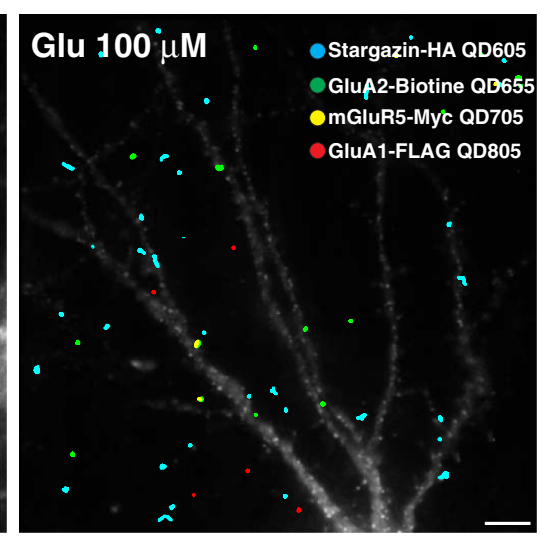

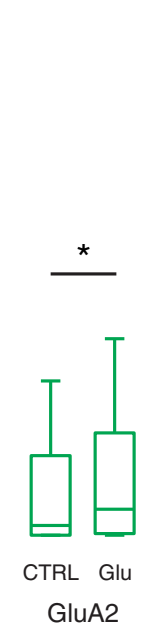

(c) CTRL

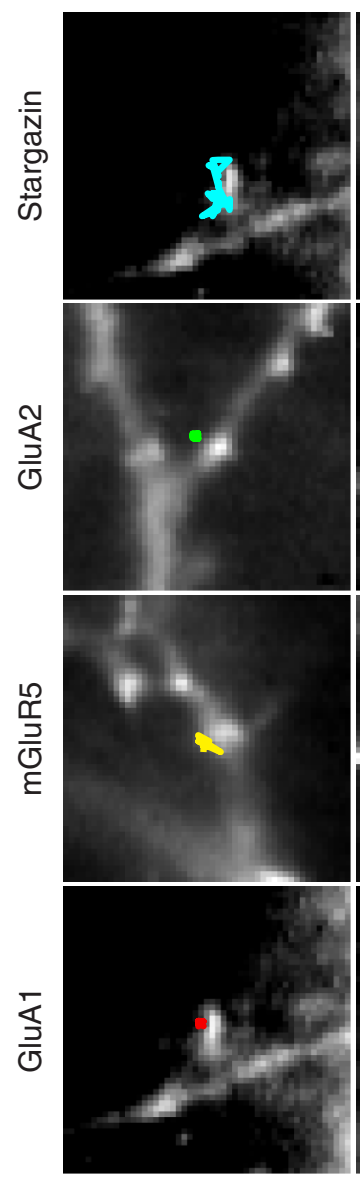

Glutamate

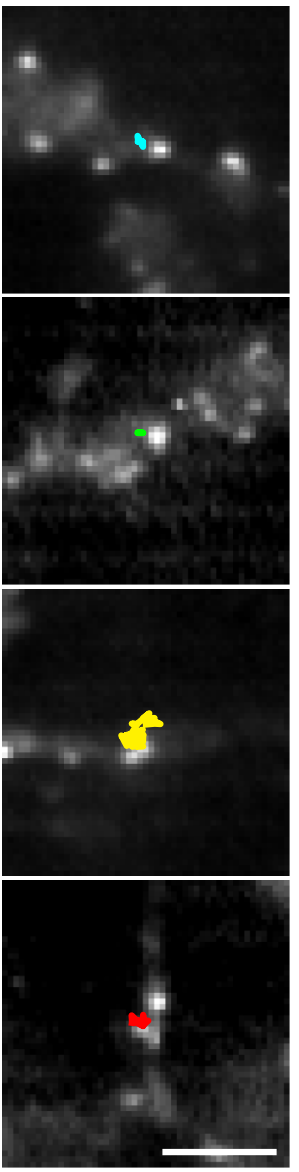

Fig. 8 (a) Example of neurons expressing GFP-CaMKII and labeled with four QDs before or following glutamate treatment, with color-coded QD trajectories over $5 \mathrm{~min}$. Scale bar $10 \mu \mathrm{m}$. (b) Diffusion coefficient of membrane receptors before and after treatment. Data are shown as median \pm IQR ( $5 \%$ to $95 \%)$. Stargazin Ctrl: $n=394, D=1.39 \times 10^{-3}$; Stargazin Glu: $n=536, D=5.80 \times 10^{-4}$; GluA2 Ctrl $n=192$, $D=4.09 \times 10^{-4}$; GluA2 Glu: $n=209, D=1.00 \times 10^{-2}$; mGluR5 Ctrl: $n=200, D=7.18 \times 10^{-4}$; mGluR5 Glu: $n=148, D=5.90 \times 10^{-4}$; GluA1 Ctrl: $n=111, D=1.90 x^{-4}$; GluA1 Glu: $n=82$, $D=2.06 \times 10^{4}$. (c) Example of synaptic diffusion of the different receptors before and after treatment. Scale bar $2.5 \mu \mathrm{m}$.

GluA2 was increased, while the mobility of mGluR5 and GluA1 was not significantly changed (Fig. 8).

\section{Discussion and Conclusion}

We assessed a hyperspectral imaging technique for single-molecule detection and tracking of multiple neurotransmitter receptors on live neuronal cells. To that end, we developed a fast hyperspectral imager - the BTF system - that allows the acquisition of full spectra of 500 to $800 \mathrm{~nm}$ in less than $3.5 \mathrm{~s}$.

We evaluated the optical performance of the BTF that offers a trade-off between already available hyperspectral technologies. Table 1 summarizes those properties. It is important to note that the AOTF, TFTF, and BTF properties were obtained while being connected to a microscope platform; this was however not the case with the LCTF.

The BTF higher transmission, compared with AOTF or LCTF, enables imaging of single QDs and the fast full spectrum acquisition permits simultaneous tracking of multiple QDs. The BTF also features acquisition of high-resolution spectra (2-nm step) of single QDs, which allows measuring their individual spectral properties. This may be useful in future experiments where specific detection of single probes can be based on their spectrum rather than on their intensity. For example, nanoprobes could report, via a spectral shift, a dynamic change inside a cell subcompartment following a specific treatment.

We combined the use of antibodies, QDs, and plasmids to track, with a high specificity, membrane receptors subtypes on live neurons. We followed the single-molecule dynamics of four receptor subtypes, while imaging a GFP-tagged synaptic protein (CaMKII). Given the spectrum resolution power of the BTF, the number of QDs with different emission peaks that could be imaged simultaneously can be much higher. The limitation on the number of probes being monitored together depends on the capability to functionalize each one with a unique tag (antibody, toxin, tag, peptide, and so on) that will specifically recognize a target of interest on the cell.

A limitation of hyperspectral imaging is the low light-transmission rate for each wavelength, rendering more difficult its application to single molecules. The total number of photons detected in a single QD PSF was on the order of 100 to 150 
Table 1 Optical properties of four hyperspectral imaging systems.

\begin{tabular}{|c|c|c|c|c|c|c|c|c|}
\hline $\begin{array}{l}\text { Imaging } \\
\text { system }\end{array}$ & $\begin{array}{l}\text { Values measured } \\
\text { when attached } \\
\text { to a microscope }\end{array}$ & $\begin{array}{c}\text { Transmission } \\
(\%)\end{array}$ & $\begin{array}{l}\text { Tuning range } \\
(\mathrm{nm})\end{array}$ & $\begin{array}{l}\text { Bandwidth } \\
(\mathrm{nm})\end{array}$ & $\begin{array}{l}\text { Tuning speed } \\
\text { (ms) }\end{array}$ & $\begin{array}{l}\text { Full spectrum } \\
\text { acquisition } \\
\text { (500 ms/image, } \\
5 \mathrm{~nm} \text { step size) (s) }\end{array}$ & $\begin{array}{l}\text { Out of band } \\
\text { rejection (OD) }\end{array}$ & Reference \\
\hline AOTF & Yes & 25 & 450 to 900 & 1.5 to 3.5 & $<0.1$ & 30 & 3 to 4 & 3 \\
\hline LCTF & No & $\begin{array}{c}5 \text { to } 33(425 \text { to } \\
725 \mathrm{~nm})\end{array}$ & 420 to 730 & 35 & 5 to 40 & N.A. & $\mathrm{OD}>2$ & $\begin{array}{c}\text { Thorlabs/Perkin } \\
\text { Elmer spec. }\end{array}$ \\
\hline TFTF & Yes & 90 & 400 to 900 & 15 to 20 & 50 to 100 & 126 & 5 & 3; Semrock \\
\hline BTF & Yes & 70 & 500 to 900 & 2 & 60 & 34 & $O D \geq 3$ & \\
\hline
\end{tabular}

photons, thereby limiting the localization error usually associated with these optical techniques. Nevertheless, the low background signal provided by the Nüvü camera allowed for subpixel accuracy for the single QD tracking.

This approach demonstrated the ability to detect simultaneously four subtypes of receptors at synapses, to track their movements at the single-molecule level, and to apply external treatments to investigate changes in their mobility. It also demonstrated the capability to combine hyperspectral imaging with quantitative analysis methods for tracking synaptic proteins in live neurons. Thus, this hyperspectral imaging system could test various compounds in a drug screening assay on several targets at the same time, which, e.g., could be applicable to pathologies of the central nervous system.

\section{Acknowledgments}

This work was supported by the Quebec Consortium for Drug Discovery (CQDM) and the Natural Sciences and Engineering Research Council of Canada (NSERC). We would like to thank Francine Nault for neuronal culture and excellent technical assistance, Isabelle Labonté and the IUSMQ Molecular Tool Platform for the GluA1-FLAG construction, Etienne LabrieDion for preliminary experiments, and Gilles Charpenet for revising the manuscript.

\section{References}

1. A. Triller and D. Choquet, "Surface trafficking of receptors between synaptic and extrasynaptic membranes: and yet they do move!," Trends Neurosci. 28(3), 133-139 (2005).

2. L. Gao and R. T. Smith, "Optical hyperspectral imaging in microscopy and spectroscopy-a review of data acquisition," J. Biophotonics 5(1), 67-84 (2014).

3. P. Favreau et al., "Thin-film tunable filters for hyperspectral fluorescence microscopy," J. Biomed. Opt. 19(1), 011017 (2014).

4. Z. Nie et al., "Hyperspectral fluorescence lifetime imaging for optical biopsy," J. Biomed. Opt. 18(9), 096001 (2013).

5. M. S. Chin et al., "Hyperspectral imaging for early detection of oxygenation and perfusion changes in irradiated skin," J. Biomed. Opt. 17(2), 026010 (2012).

6. A. D. Elliott et al., "Real-time hyperspectral fluorescence imaging of pancreatic beta-cell dynamics with the image mapping spectrometer," J. Cell. Sci. 125(Pt 20), 4833-4840 (2012).

7. S. J. Leavesley et al., "Hyperspectral imaging microscopy for identification and quantitative analysis of fluorescently-labeled cells in highly autofluorescent tissue," J. Biophotonics 5(1), 67-84 (2012).

8. P. J. Cutler et al., "Multi-color quantum dot tracking using a high-speed hyperspectral line-scanning microscope," PLoS One 8(5), e64320 (2013).
9. S. Blais-Ouellette, O. Daigle, and K. Taylor, "The imaging Bragg tunable filter: a new path to integral field spectroscopy and narrow band imaging," Proc. SPIE 6269, 62695H (2006).

10. M. Heine et al., "Surface mobility of postsynaptic AMPARs tunes synaptic transmission," Science 320(5873), 201-205 (2008).

11. P. Opazo et al., "CaMKII triggers the diffusional trapping of surface AMPARs through phosphorylation of stargazin," Neuron 67(2), 239252 (2010).

12. H. Kogelnik, "Coupled wave theory for thick hologram gratings," Bell Syst. Tech. J. 48(9), 2909-2947 (1969).

13. M. Howarth et al., "Targeting quantum dots to surface proteins in living cells with biotin ligase," Proc. Natl. Acad. Sci. U.S.A. 102(21), 75837588 (2005).

14. A. Yildiz et al., "Myosin V walks hand-over-hand: single fluorophore imaging with 1.5-nm localization," Science 300(5628), 2061-2065 (2003).

Simon Labrecque is an optical engineer and neuroscientist. He received an $\mathrm{MSc}$ in holography and a $\mathrm{PhD}$ in biophotonics from Université Laval. He has a strong interest in live cell microscopy, new development in optical microscopy, and single-molecule tracking with fluorescent molecules or nanoparticles for applications in neurosciences. He is currently at the Centre de recherche de l'Institut universitaire en santé mentale de Québec. He is the coordinator of novel imaging approaches for Brain Canada's Neurophotonics platform.

Jean-Philippe Sylvestre obtained his PhD in pharmaceutical sciences after completing his degree and his MSc in engineering physics. He joined Photon Etc. in 2011 as a scientist to lead the development of hyperspectral imaging platforms for biomedical applications. He is now CTO at the Optina Diagnostics, where he manages the development of noninvasive optical diagnostic tools for diseases having manifestations in the retina.

Stephane Marcet is an optical engineer. He obtained his PhD in physics from Joseph Fourier University, Grenoble, France. He is currently a researcher in optics at Photon Etc., a company providing scientific imaging solutions. He is specialized in microscopy and in the development of new hyperspectral imaging systems.

Francesca Mangiarini holds an MSc in industrial catalysis from the University of Milan and a PhD in chemistry from Concordia University, Canada, where she worked on the synthesis and characterization of upconverting nanoparticles. She works as application scientist at Photon Etc. and is in charge of the development of hyperspectral microscopy platforms to image nanomaterials for life sciences.

Brice Bourgoin is an electrical engineer with a master's degree in automated manufacturing engineering from the École de Technologie Supérieure, Montréal, Canada. He works as an artificial vision expert at Photon Etc., a company that provides scientific imaging solutions for a vast array of applications. He specializes in data processing, algorithms, calibration, and software integration.

Marc Verhaegen obtained his master's degree in optoelectronics from the Université de Liège in Belgium and his PhD in physics 
from the Université de Montréal. He completed his postdoctoral studies in the fiber-optics laboratory at the École Polytechnique de Montréal, working on the intensification of second-order nonlinear effects for fiber-optics and silica glass. He has worked as a researcher in the R\&D departments of companies including ITF Optical Technologies and Corvis Canada.

Sébastien Blais-Ouellette is the president and founder of Photon Etc. While working at the Astronomy Department of California Institute of Technology, Caltech, he perfected a technology that uses volume Bragg gratings to combine images and spectra of an object or sample. After obtaining a master's degree in Montreal and a PhD at the Université de Montréal and the Université d'AixMarseille in France, he worked as a researcher at the Lawrence Livermore National Laboratory and Caltech.

Paul De Koninck, $\mathrm{PhD}$ in neurobiology, is a professor of biochemistry, microbiology, and bio-informatics at Université Laval, the director of the Biophotonics Graduate Program at Université Laval, and the director of the Cellular and Molecular Division at the Centre de recherche de l'Institut universitaire en santé mentale de Québec. His research interests are on exploiting and developing optical methods to investigate the mechanisms of synaptic signaling and remodeling, related to learning and memory. 